\title{
ARTICLE OPEN \\ Adhesive behavior and detachment mechanisms of bacterial amyloid nanofibers
}

\author{
Ao Wang ${ }^{1}$ and Sinan Keten (iD) ${ }^{1,2}$
}

\begin{abstract}
Amyloid nanofibers, such as curli nanofibers, have proven capable of adhering strongly to abiotic surfaces. However, the adhesive performance of individual nanofibers and the dependence of this performance on physical properties remain to be characterized. We carried out coarse-grained molecular dynamics simulations to determine the detachment mechanisms of single amyloid fibers from surfaces. Taking a generic model inspired from the curli nanofiber subunit CsgA, we discover that the amyloid nanofibers can undergo three different peeling processes when pulled at a constant rate normal to the surface. Computational phase diagrams built from parametric studies indicate that strong nanofibers with high cohesive energy detach by peeling smoothly away from the substrate while weak fibers break prematurely. At intermediate ratios, hinge formation occurs and the work of peeling the nanofiber is twice the adhesive energy due to the additional energy required to bend the nanofiber during desorption. Varying the geometry of amyloid subunits revealed that the work of peeling decreases for thicker nanofibers, suggesting that the tape-like monomeric structure of amyloids may facilitate better adhesive performance. Our results demonstrate how the dimensions and adhesive and cohesive properties of the amyloid nanofibers can be optimized to resist mechanical peeling.
\end{abstract}

npj Computational Materials (2019)5:29; https://doi.org/10.1038/s41524-019-0154-7

\section{INTRODUCTION}

Since their discovery, bacterial amyloids have been detected in many microorganisms, serving various purposes ranging from virulent to structural. ${ }^{1}$ Many surface-associated amyloid structures take part in biofilm extracellular matrices, including curli, FapC, TasA, PSM, Adhesin P1 and others, indicating their production may be common among microorganisms that form biofilm. ${ }^{1}$ In addition to providing structure to biofilms, amyloid nanofibers, such as curli fibers, also participate in the initial attachment of cells to surfaces, ${ }^{2}$ host cell invasion, ${ }^{3,4}$ binds to human contact system proteins, $^{5-7}$ and facilitates adhesion to surfaces. ${ }^{8,9}$ Synthetic amyloid nanofibers also exhibit strong adhesive properties both to organic and inorganic surfaces. ${ }^{10-14}$ The adhesive capabilities of amyloid nanofobers mark them as a potential bioadhesive ingredient.

Bioinspired and biological adhesives are highly desired in applications where biocompatibility and wet adhesion are important. Strong adhesion typically requires specific or covalent binding to surfaces combined with cohesive energy dissipation mechanisms in the adhesive film. This strategy has been instrumental in creating adhesive hydrogels can achieve great strength and toughness while requiring significant energy dissipation for detachment when they are chemically anchored to surfaces. ${ }^{15,16}$ The strong adhesion of mussel feet and barnacle cement has attracted research interest for biomimicry, and synthetic biomimetic polymers have already achieved great adhesive strength by utilizing DOPA in polymer-based glues. ${ }^{17,18}$ Gels and biofilms facilitating amyloids such as curli fibers can now be produced in an accessible, scalable approach using filtration. ${ }^{19}$ This framework may be useful for creating strong adhesives owing to the ease of self-assembly, flexural rigidity, ${ }^{20}$ and network forming ability of functional amyloids. ${ }^{21}$

Taking curli as an example, the curli major subunit CsgA polymerizes on the surface of many phyla of bacteria (including $E$, coli) ${ }^{1}$ and also self-assembles readily into long, semi-flexible nanofibers, which can be tailored for different functions through point mutations, peptide fusions, and conjugates. Modifications so far have included conjugations to enhance wet adhesive strength, ${ }^{13,14}$ fusion proteins, ${ }^{13,22}$ electronic conduction, ${ }^{23}$ and more. Multiple curli mutant studies have observed introduction of variations in the subunit structure can alter morphology and mechanics. ${ }^{13,22}$ Although many types of modifications have been successfully applied to curli, the mechanical details of the native system are still not well understood.

So far, the curli system has been well-studied in the context of amyloid formation, ${ }^{24,25}$ but mechanical details of the curli system and other bacterial amyloids-especially at submicron resolution -have been limited. Recently, the growth of single curli fibers has been examined in great resolution using atomic force microscope (AFM) ${ }^{26}$ revealing details of growth dynamics. The binding of single curli monomers and fibers to fibronectin has also been characterized using AFM, finding clusters of bonds facilitating in the binding and unbinding events. ${ }^{27}$ As experiments have moved toward mechanically studying curli at smaller length scales, modeling approaches have taken a bottom-up direction. The structures of CsgA subunits derived from molecular modeling approaches have been employed to study curli mechanics and adhesion. ${ }^{28,29}$ The adhesion of single CsgA monomers to a surface has revealed the importance of flexibility and side chain chemistry, as well as correlated motion within the curli core. ${ }^{30}$ Coarse-grained (CG) simulations of nanofibers using steered molecular dynamics

\footnotetext{
${ }^{1}$ Department of Civil and Environmental Engineering, Northwestern University, Evanston, IL 60208, USA and ${ }^{2}$ Department of Mechanical Engineering and Environmental Engineering, Northwestern University, Evanston, IL 60208, USA

Correspondence: Sinan Keten (s-keten@northwestern.edu)
}

Received: 7 August 2018 Accepted: 8 January 2019

Published online: 01 March 2019 

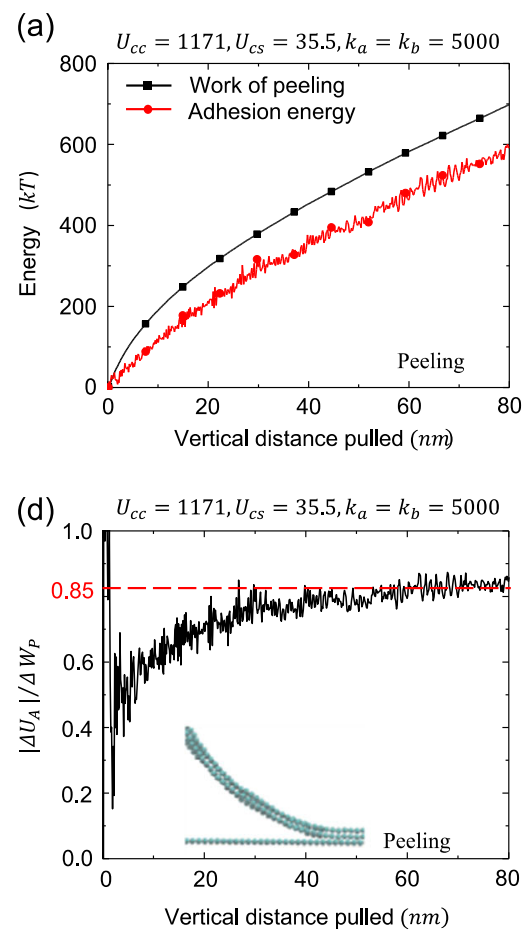
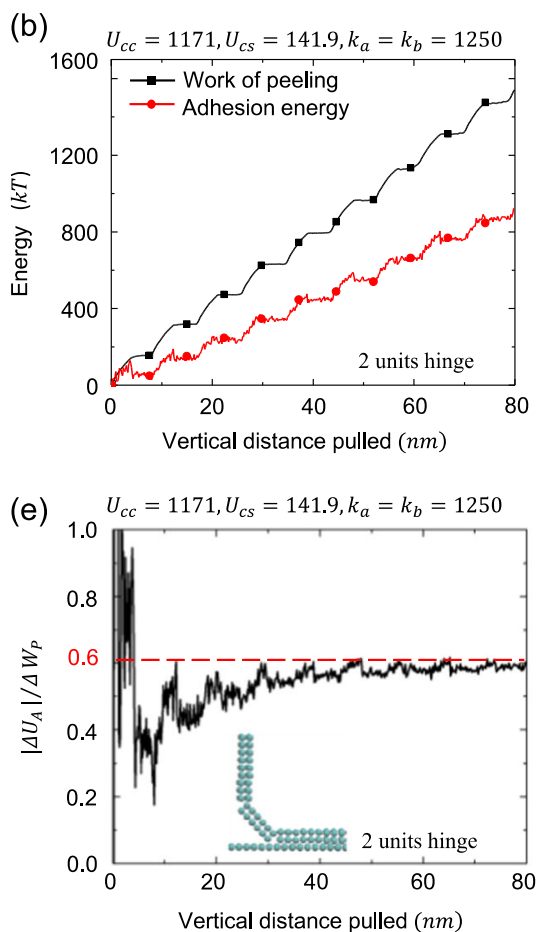
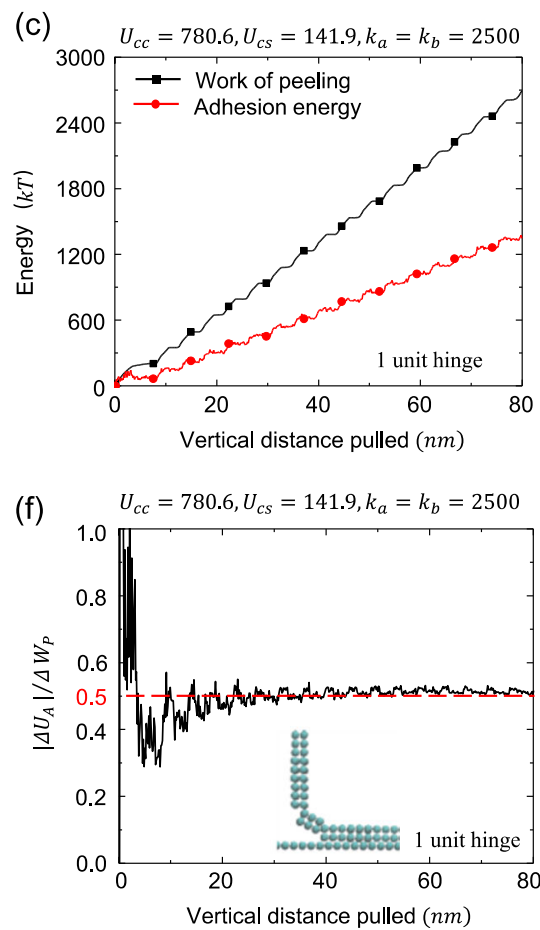

Fig. 1 a-c The total work of peeling (black solid lines) and the adhesive energy (red solid lines) between the nanofiber and the substrate with respect to the vertical peeling distance under the "peeling" and the "hinge" modes. $\mathbf{d}-\mathbf{f}$ The black lines represent the ratio of dissipated adhesive energy over accumulated work of peeling $\left(\left|\Delta U_{A}\right| / \Delta W_{P}\right)$ with respect to the vertical peeling distance $(\mathrm{y})$. The red dashed lines represent the steady state values. The snapshots are the conformations of the peeled nanofibers under different modes obtained from CG-MD simulation. In these figures, the energy unit is $k T$ and the spring constant unit is $\mathrm{kT} / \mathrm{nm}^{2}$

have shown the importance of bending rigidity in resisting desorption. $^{31}$

While it has now been demonstrated that it is possible to isolate and modify amyloid nanofibers and form useful materials from these building blocks, there has not yet been a systematic study of the influence of fiber dimensions, adhesive and cohesive properties on their adhesive performance. To address this issue and provide guidance to future bioengineered amyloid nanofiber systems, here we examine the influence of geometric and energetic properties in the failure behavior of model amyloid nanofibers during desorption. Building on prior coarse-grained studies, $^{31}$ here we provide a comprehensive investigation of the parameters governing peeling behavior, including adhesive and cohesive energy of subunits, nanofiber length and thickness, as well as subunit rigidity.

\section{RESULTS AND DISCUSSION}

We first present results from peeling simulations where adhesive and cohesive energy parameters, Ucs and Ucc respectively, were varied. A systematic sweep involving these two parameters revealed that the nanofiber might undergo one of three different detachment processes, which we identify as "peeling", "hinge", and "break" modes. As the name implies, in "peeling", the nanofiber peeled smoothly and continuously away from the substrate, exhibiting no discontinuities in curvature and with the nanofiber remaining intact (Fig. 1d). In the "break" mode, the nanofiber ruptured, as it cannot withstand the bending or tensile forces. In the "hinge" mode, after several units were peeled away from the substrate, the nanofiber formed a region with a small radius of curvature, and this localization lead to a "hinge mechanism" at the interface of adjacent subunits, making the several units rotate around the upper beads of the posterior subunit with little resistance (Fig. 1e, d).
In the hinge mode, we further discovered that the number of units peeled concurrently between subsequent hinge formations could differ. Several sizes between 1-10 units were observed, but here we focus in "1-unit" and "2-units" hinge cases. Figure 1a-c show the total work of peeling and the adhesive energy between the nanofiber and the substrate with respect to the peeling distance under "peeling" and the "hinge" modes. The adhesive energy is the change in the sum of the attractive $12 / 6 \mathrm{LJ}$ potential energies between the nanofiber and the substrate beads. Specifically, we are measuring the change in adhesive energy | $\Delta U_{A}$ | to quantify the enthalpic, non-bonded energy contribution to the work of peeling, $\Delta W_{P}$, calculated from SMD simulation. The slope of the work of peeling at the beginning is larger than that of the steady state. This results from the fact that the moment induced by the force has to be relatively large at the beginning of the peeling process to induce bending in the nanofiber, leading to larger work of peeling in this transient regime. ${ }^{32-34}$ During the two "hinge" peeling processes, the work of peeling has short plateau regions, corresponding to the formation of hinges and rotations along them before peeling progresses further. We further calculated and plotted the ratio of adhesive energy to work of peeling $\left(\left|\Delta U_{A}\right| / \Delta W_{P}\right)$ with respect to the peeling distance (y) (Fig. $1 \mathrm{~d}-\mathrm{f}$ ) for three representative cases. In all three cases presented, at the beginning of peeling, the ratio almost drops to zero. When the peeling process fully develops, the ratio stays around a certain value between 0 to 1 . In the "peeling" case, $80 \%$ of the total peeling work is the cost of overcoming the adhesive energy; while in the two "hinge" cases, more energy is dissipated by sources other than adhesive energy. One main reason is that when there are hinges, the circumstance of the peeling front is similar to that of the beginning of the peeling process, requiring larger moment of force to locally bend the unit following the peeling front. The ratio of the " 1 -unit hinge" case is about $50 \%$, which is smaller than that of the "2-units hinge" case, verifying the fact that coordinated separation of multiple subunits will make the nanofiber more 
(a) Phase diagram of fibril's peeling behavior

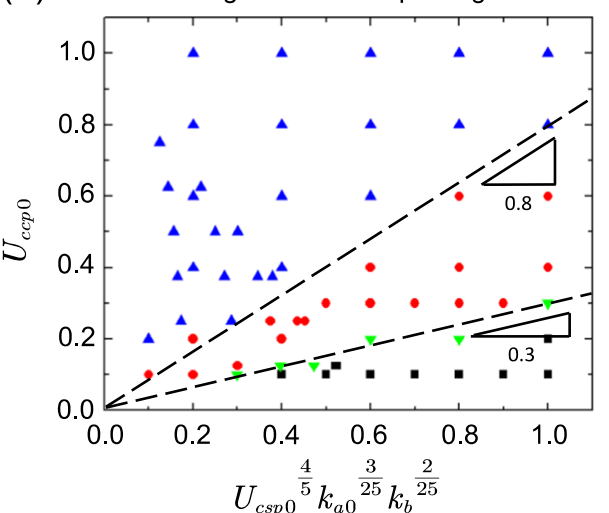

(b)

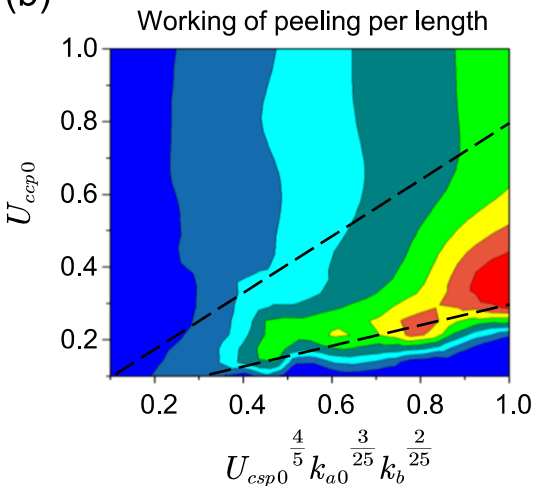

- Break mode

$\checkmark$ Hinge mode (1 unit)

- Hinge mode

$\Delta$ Peeling mode
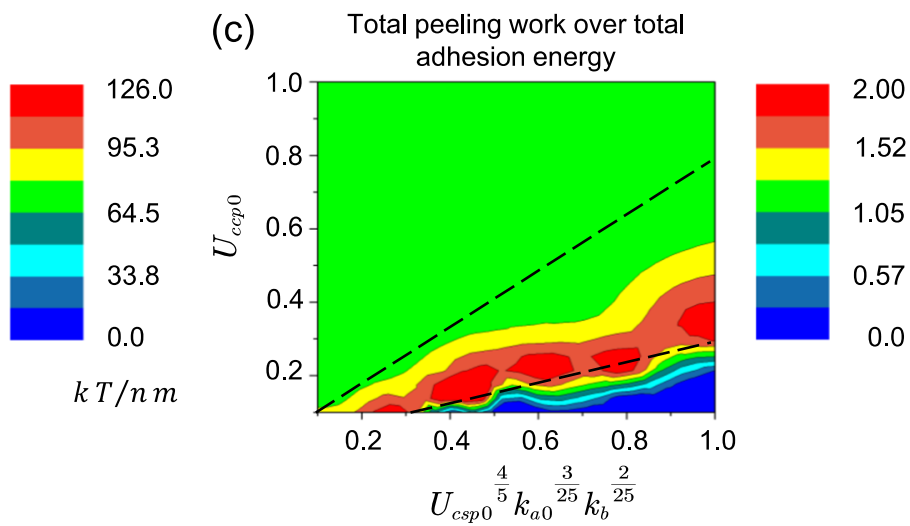

Fig. 2 a The phase diagram of the detachment behavior of the fiber. There are three main modes of detachment, the "break" mode, the "hinge" mode and the "peeling mode", which are separated by two dashed lines. b The color map quantifies the work per length required to detach the fiber from the substrate $\left(W_{P} / L\right)$ (units $\mathrm{kT} / \mathrm{nm}$ ). c The color map quantifies the ratio of the work of detachment over the total adhesive energy between the fiber and the substrate $(\eta)$. This figure illustrates that it is possible to roughly double the work required to detach a fiber when the hinge mode is activated

difficult to deform and lead to less contribution from the bending energy. The specific ratio of $50 \%$ merits further elaboration.

In continuum mechanics, peeling an elastic or viscoelastic strip from the substrate is widely studied. ${ }^{32-43}$ When the substrate is highly adhesive, the peeling front of the strip will have a very small radius of curvature, meaning that the maximum principal strain and maximum principal stress are localized in this region and are larger than the rest of the nanofiber. The local material will store extra elastic energy comparable to the local adhesive energy. Furthermore, if the elastic strip is not extensible and is peeled steadily in the vertical direction, the total work of peeling is expected to be twice the total adhesive energy between the strip and substrate, ${ }^{31,44,45}$ which matches the "1-unit hinge" case. This tells us the importance of local curvature to maximize the total peeling work. Different from a continuum elastic strip model, the discrete nature of subunits making up the amyloid nanofiber results in hinge formation to accommodate the local curvature.

Next, we conducted systematic simulations to investigate more broadly the main factors that govern the mechanisms and work of peeling of the coarse-grained amyloid nanofibers from the substrate. We examined four parameters, the cohesive energy per area between two subunits $\left(U_{c c p}\right)$, the adhesive energy per area between one subunit and the substrate $\left(U_{c s p}\right)$, the bond elasticity coefficient $\left(k_{b}\right)$ and the angle elasticity coefficient $\left(k_{a}\right)$. These parameters are all expressed in terms of $\mathrm{kT} / \mathrm{nm}^{2}$ as units. The values of the parameters are tuned to reflect the strong and weak cohesive interfaces between nanofiber subunits and the different interfacial energies between subunits and the substrate. The elasticity parameters confer the stiffness and rigidity of the subunits themselves, which we assume should require large tensile forces to unfold. After various trials, we selected that the maximal value of $U_{c c p}$ was $390.3 \mathrm{kT} / \mathrm{nm}^{2}$; the maximal value of $U_{\text {csp }}$ was $14.2 \mathrm{kT} / \mathrm{nm}^{2}$ and the maximal values of the bond and angle elasticity coefficients $\left(k_{b}, k_{a}\right)$ were $10,000 \mathrm{kT} / \mathrm{nm}^{2}$. At the lower end of the parameter space, we chose the minimal values of the bond and angle elasticity coefficients to be $100 \mathrm{kT} / \mathrm{nm}^{2}$. For these lower bound values, the Young's modulus is $0.14 \mathrm{GPa}$, which is one order of magnitude less than the lower bound of values reported for amyloid nanofibers. ${ }^{46}$ The overall parametric range is fairly broad and should cover a variety of scenarios attainable with biological and engineered amyloid nanofibers. For simplicity, we normalized the parameters by their maximal values in reporting the results; hence, values ranged from 0 to 1 . Before going to further discussion, we review the influences of these parameters first. There are comprehensive studies which theoretically and numerically analyze the process of peeling an isotropic and linearly elastic 2D strip or sheet from a flat and rigid substrate. $^{32,35}$ Under pure peeling, the local bending energy density, which governs the additional detachment penalty, is proportional to the composite variable $\sqrt{\gamma E I}$, where $\gamma$ is the interfacial energy density between the 2D sheet and the substrate, and EI is the out-of-plane bending stiffness, ${ }^{35}$ which is proportional to the persistence length of amyloid nanofibers. ${ }^{47}$ The key take away from this analysis is that both the adhesive energy, as well as the persistence length explicitly play a role on the detachment mechanism of an amyloid nanofiber from the surface. The work carried out in the process also depends on these two variables. 

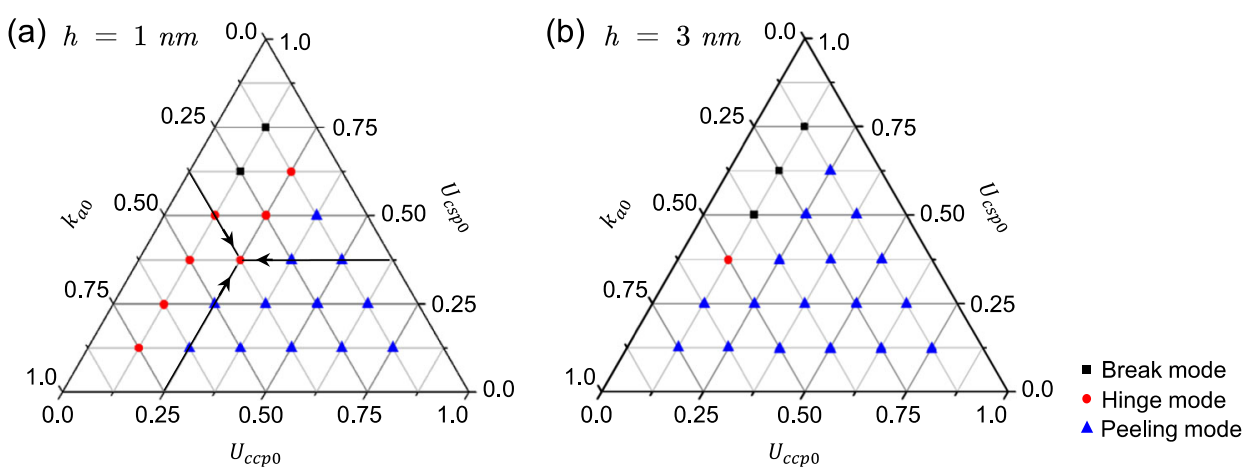

Fig. 3 a, b The phase diagram of the detachment behavior of the nanofibers with different heights, $1 \mathrm{~nm}$ and $3 \mathrm{~nm}$, respectively. The figure illustrates that thicker nanofibers transit towards peeling modes, whereas thin nanofibers can exhibit strong adhesion enabled by a transition into the hinge mode when adhesive and cohesive interactions are tailored in the right range
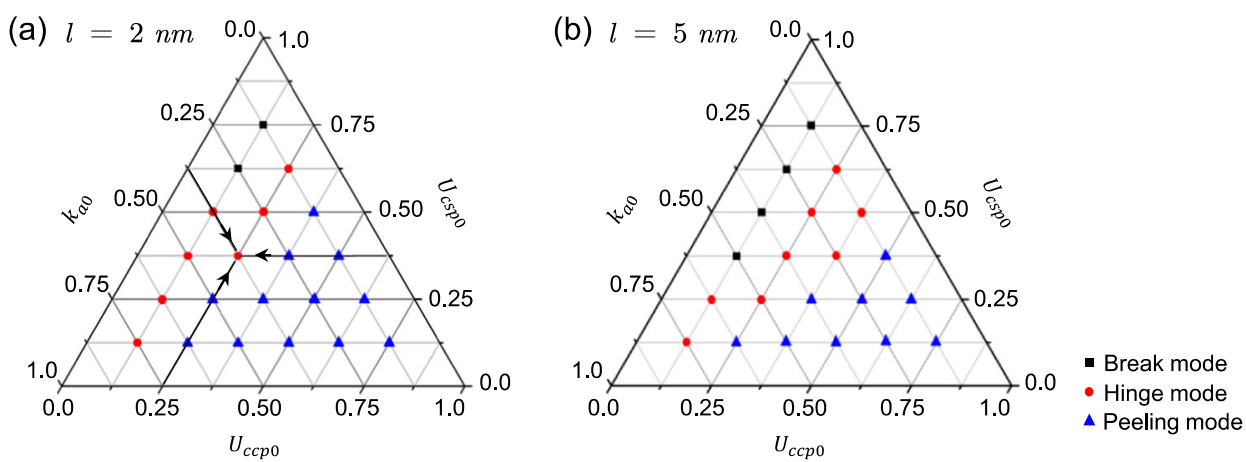

Fig. 4 a, b The phase diagram of the detachment behavior of the nanofibers with different lengths, $2 \mathrm{~nm}$ and $5 \mathrm{~nm}$, respectively. The figure illustrates that when the subunit length decreases, the nanofibers transit towards peeling modes

Based on this analysis, we merged the four parameters into two composite variables. Meanwhile, the four parameters are all normalized to 1 through dividing them by their maximal values. Figure $2 a$ shows the phase diagram obtained for nanofiber detachment using these two variables. The $y$-axis parameter is the normalized cohesive energy per unit area $\left(U_{c c p 0}\right)$; the $x$-axis parameter is the composite variable $\left(U_{c s p 0}^{\frac{4}{5}} k_{a 0}^{\frac{3}{25}} k_{b 0}^{\frac{2}{25}}\right)$, containing the normalized adhesive energy per unit area $\left(U_{s c p 0}\right)$ and the normalized bond and angle elasticity coefficients $\left(k_{b 0}, k_{a 0}\right)$. The value of the superscripts, determined empirically here to classify the data most effectively (also add up to 1), indicate that the cohesive energy per area and the adhesive energy per area are more pivotal parameters in determining the behavior of peeling process. Due to the pure peeling conditions imposed here, where the nanofiber is pulled normal to the surface, the bending rigidity plays a more important role than the axial stiffness of the nanofibers. $^{32,35}$ In Fig. 2a, we could observe that there are three regions divided by two dashed lines originating from the origin. The upper region stands for the "peeling" mode; the middle region covers the "hinge" mode; and the bottom region represents the "break" mode. The slopes of the two dashed lines are 0.8 and 0.3 , respectively. The upper dashed line marks the transition from the "peeling" mode to the "hinge" mode; while the bottom dashed line indicates the transition from the "hinge" mode to the "break" mode. Notably, the "1-unit hinge" cases happen just around the bottom line. Figure $2 b$ is the color map of the total work of peeling per length $\left(W_{P} / L\right)$. The values of the "break" cases are always very small. The values of the "peeling" cases are in the mid-range. At any given value on the $x$-axis, that is, changing only the cohesive energy of the nanofiber, we can observe that attaining the "hinge" mode yields the largest amount of work required to peel the nanofiber. The largest $W_{P}$ locates in the right part of the phase diagram, near the bottom dashed line. In experiments, the adhesion energies per length of amyloid nanofiber at liquid-liquid interfaces were estimated $\sim 60 \mathrm{kT} / \mathrm{nm},{ }^{48}$ which means $W_{P} / L$ (larger than the adhesive energy per length) lies almost in the hinge-formation area. Figure $2 \mathrm{c}$ plots the color map of the ratio of the work of peeling over the total adhesive energy between the nanofiber and the substrate $(\eta)$. In the bottom region, $\eta$ is near zero, which means that effective adhesive performance cannot be achieved when the fibers are detaching in the "break" mode. In the top region, $\eta$ is about 1 , indicating that the adhesive energy dominates peeling process. In the middle region between the two lines, $\eta$ reaches its maximum value around 2 near the bottom line, matching the continuum limit for small vanishing radius of curvature. It is clear from this curve that the optimal case is peeling with a high curvature on the verge of breaking, any reduction in the adhesive energy or reduction in the cohesive energy beyond this point sharply reduces the work of peeling as indicated by the sharp color gradient below the lower dashed line.

Above simulations are all about the fiber consisting of 30 flat subunits, with the height, width and length being $1 \mathrm{~nm}, 3 \mathrm{~nm}$, and $2 \mathrm{~nm}$, respectively. Actually, in vivo, most of the functional amyloid nanofibers have this strip-like conformation in nanoscale. ${ }^{29,49-51}$ Motivated by this observation, we first examine the effects of subunit height, $h$, on the adhesive behavior. To find the influence of subunit height or equivalently nanofiber thickness, we modified our model by increasing the height of the subunits, $h$, with other parameters unchanged. Figure $3 a$, $b$ show the phase diagram of nanofiber detachment, for nanofibers with different heights, $1 \mathrm{~nm}$, $3 \mathrm{~nm}$, respectively. The values of the bond coefficients are set the same as those of the angle coefficients in this scenario. Accompanied with the increase of the height, many cases in the "hinge" mode transitioned to the "peeling" mode, resulting in an 

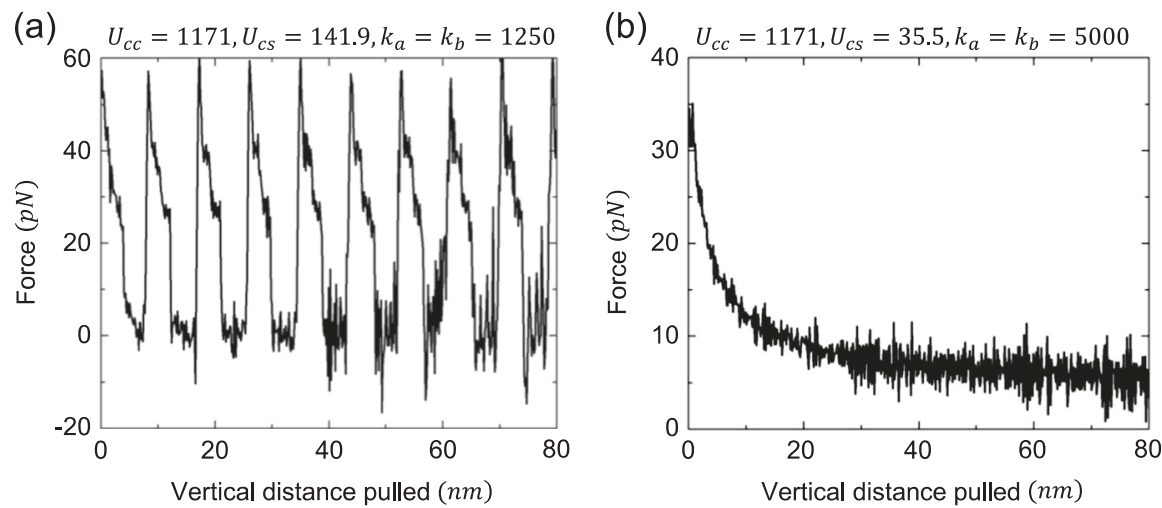

Fig. 5 a One representative curve of peeling force with respect to the vertical peeling distance under the "2-units" hinge case. The force curve displays "saw-tooth" like shape. The peaks of the curve correspond to the formation of hinges; and the valleys of the curve correspond to the detachment process. b One representative curve of peeling force with respect to the vertical peeling distance under the "peeling" case. In these figures, the energy unit is $k T$ and the spring constant unit is $\mathrm{kT} / \mathrm{nm}^{2}$

almost binary phase diagram. Thicker nanofibers have larger cohesive energy per subunit's width. Together with the larger bending rigidity, this prevents the fracture of the interface, hence limiting the possible "hinge" cases.

It is known that misfolded short protein segments can also selfassemble into insoluble amyloid nanofibers, ${ }^{52}$ with only one betastrand representing a subunit subunit. ${ }^{53}$ These nanofibers are associated with diverse neurodegenerative diseases. Conversely, functional bacterial amyloids such as curli have discrete subunits that employ multiple beta-strands. Noting this difference, we studied the influence of the length of the subunit on the detachment behavior of a nanofiber. Figure $4 a$, $b$ show the phase diagram of nanofiber detachment, for nanofibers with different lengths, $2 \mathrm{~nm}, 5 \mathrm{~nm}$, which are the original case and a longer subunit case, respectively. With the longer subunit length, three cases transition from the "peeling" mode to the "hinge" mode, and two cases transition from the "hinge" mode to the "break" mode. This informs us that nanofibers with shorter subunits, all else being the same, are less prone to breakage. This is because the overall bending rigidity of nanofibers are expected to be higher when the subunit length is greater, considering that in our model the subunits have relatively high stiffness compared to interfaces. This effect should diminish as the properties of the subunits approach that of the interface between subunits, i.e., when the interfaces are not particularly weaker.

Now that we have established key factors governing the mechanism of detachment, the next question we wish to address is how the detachment mechanism influences the force response of the nanofiber. Figure 5 shows the peeling force with respect to the peeling distance. The curve is "saw-tooth" like for the hinge mechanism. The peaks of the curve represent the hinge formation processes; while the steep and then gentle dropping of the force curves represent the continuous detachment of the subsequent subunits. These sawtooth features disappear when pure peeling is occurring. Typical peeling of protein-based nanofibers from surfaces involve saw-tooth like force response curves which are attributed to local unfolding events. This observation is interesting because here we see that even without unfolding, saw-tooth curves, albeit having a different characteristic, can occur due to hinge formation. Whether cooperative detachment of multiple subunits via hinge formation is relevant for the detachment behavior of the functional amyloid nanofibers is not known yet. Future studies can consider the possibility of subunit unfolding, which is simply another mode of strain localization, to ascertain different mechanisms governing the force signature of amyloids. No matter which circumstance, the model presented above provides a simple way to study the peeling behavior of the functional amyloid nanofibers.
The model presented here, while simplifying the actual structure of amyloid nanofibers made from CsgA, should be broadly applicable to a wide range of supramolecular assemblies. The model could be further developed to study nanofibers other than the $E$. coli curli nanofibers, and may incorporate other features such as unfolding mechanisms. Other boundary conditions such as pulling at different angles could also be examined readily with these models, and we anticipate that cohesive energy and nanofiber elasticity may start to play a greater role as the pulling angle is reduced. Similar amyloid nanofibers may have different subunits, cross-sectional shapes, and such effects may also be studied with coarse-grained approaches. ${ }^{54-57}$ Different nanofibers may also have different mechanical properties ${ }^{58}$ and it would also be interesting to see the effects of modifying the mechanical properties of nanofibers on their detachment behavior. For instance, mutations and grafts on the peptide sequence of functional amyloids such as curli can change the interfacial strength, as well as mechanical properties such as bending rigidity, which may explain variations in the performance of amyloid based adhesives. ${ }^{59-62}$ Furthermore, polymeric or DNAbased self-assembled nanofibers could also be studied in a similar manner to examine whether biological amyloids offer any advantages over such systems in terms of adhesive performance. ${ }^{63,64}$ The current work focusing on the development of computational phase diagrams for adhesion sets the stage for pursuing these diverse research directions to evaluate a variety of interfacial phenomena relevant to biological, as well as engineered adhesives. Higher scale models, made possible with further coarsening would shed light on how bundles and networks of these amyloid nanofibers respond to forces, to assess how single nanofiber properties translate into properties of the networks and biofilms formed from such systems.

In summary, we proposed a generic coarse-grained model for single amyloid nanofibers, inspired from the E. coli cross-beta protein structure called curli. The detachment behavior of single nanofibers when pulled normal to a substrate were extensively characterized here through a parametric study that looked at energetic and geometric effects. We discovered three different detachment modes, namely, the "break" mode, the "hinge" mode and the "peel" mode. We examined four parameters, the cohesive strength, the adhesion strength, the bending stiffness and the stretching stiffness; and we combined these variables to composite parameters that facilitate simpler phase diagrams. Tuning the two composite parameters pertaining to adhesion vs. cohesive or elasticity, we plotted a phase diagram to classify regions defined by the three detachment modes. Owing to the discrete nature of the subunits making up the amyloid, the competition between the nanofiber cohesive strength and the 
(a)

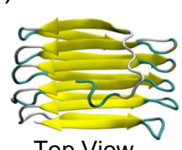

Top View

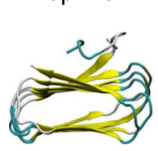

Front View

(c)

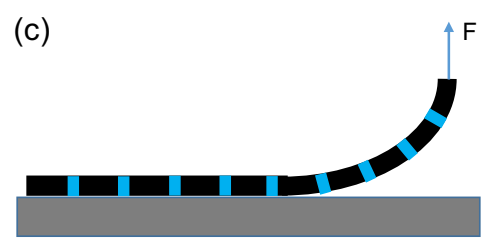

Fig. 6 a The atomistic structure of the curli subunit CsgA as a representative adhesive amyloid motif, shown here from the top view and the front view. ${ }^{29} \mathbf{b}$ Different views of the coarse-grained model of the subunit. The coarse-grained beads in the subunit are linked by harmonic bonds (black solid lines) and angular springs to provide rigidity; the vertical red dashed lines represent attractive 12/ $6 \mathrm{LJ}$ interactions and the diagonal blue dashed lines represent repulsive interactions. The subunit adheres to the substrate by $12 / 6$ $\mathrm{LJ}$ interactions. The strength of adhesive $\left(U_{\mathrm{cs}}\right)$ and cohesive $\left(U_{\mathrm{cc}}\right) \mathrm{LJ}$ interactions are parametrically studied by varying the depth of the energy well for each type of interaction. Subunit geometry effects on adhesion are examined by varying $h, l$. c The peeling setup of the simulations, showing vertical detachment of an amyloid model from a surface. The black sections represent the CsgA subunits while the blue sections represent the nonbonded interaction area

adhesion strength governs the phase diagram. Because of vertical peeling and high axial stiffness of amyloids, the bending stiffness is more important than the stretching stiffness. In the "peeling" mode, most of the work is attributed to the cost of overcoming the adhesive energy; while in the "hinge" mode, extra energy must be dissipated to bend the subsequent subunits, leading to the doubling of the ratio of the total peeling work to the adhesive energy. This matches the theoretical results of peeling a continuous strip with a small local radius of curvature. Examining the effect of nanofiber thickness, we found that due to the larger bending rigidity, thicker nanofibers depress the hinge formation mechanism. This may explain why the amyloid nanofibers have the strip-like shape, namely to achieve greater adhesion by forming flexible, adhesive, tape-like structures. Regarding to the length of the nanofibers, we found that shorter subunit lengths reduced the bending induced breakage of the nanofibers as they could accommodate sharper bends than nanofibers consisting of long, stiff links. Finally, we show that saw-tooth shaped peeling curves can occur during detachment even in the absence of unfolding, although the relevance of this mechanism to amyloids is yet to be verified experimentally. Overall, our computational analysis highlights the interplay between nanofiber strength, adhesion, geometry and flexibility on the resistance of amyloids to mechanical detachment from surfaces. These findings may guide future studies on biofilm removal from surfaces, design of biofilmbased materials, or microbial manufacturing of bio-adhesives.

\section{METHODS}

The curli subunit CsgA is a $13 \mathrm{kDa}$ protein with three domains: a signaling sequence that is cleaved, an unstructured $\mathrm{N}$-terminal domain, and a $\mathrm{C}$ terminal amyloid core. The C-terminal amyloid domain contains five imperfect repeats with a strand-loop-strand motif, aligned roughly orthogonal to the nanofiber growth direction. The beta-helical CsgA subunit forms a shape that resembles a stable rectangular slab stabilized by interstrand hydrogen bonds, as well as conserved residues in the interior. To build a representative bacterial amyloid model inspired from CsgA, here we idealize subunits as a rectangular box made up of coarsegrained beads for simplicity and generality. The initial choice of the height, width, and length of these subunits are $h=1 \mathrm{~nm}, w=3 \mathrm{~nm}, l=2 \mathrm{~nm}$, respectively, based loosely on CsgA dimensions $(h=0.96 \mathrm{~nm}, w=2.88 \mathrm{~nm}$, $I=1.92 \mathrm{~nm}){ }^{29}$ We then systematically look at the effects of the subunit dimensions. An all-atomistic structure developed for the CsgA subunit can be found in Fig. 6a, and the coarse-grained model used here is shown in Fig. $6 \mathrm{~b}$. Each nanofiber simulated contains 30 subunits. The subunits can stack end-to-end and self-assemble into the amyloid nanofiber stabilized by attractive non-bonded interactions at terminal interfaces, such as hydrophobic, aromatic, electrostatic and van der Waals interactions. ${ }^{65}$ Within one nanofiber, adjacent beads are $1 \mathrm{~nm}$ apart, and are linked by harmonic springs (the equilibrium distance is $1 \mathrm{~nm}$ ), and the vertical positions across strands are maintained by harmonic angle potentials (equilibrium angle is 90 degrees). Therefore, the stretching and bending stiffness of the subunit can be tuned by changing the stiffness of the bonding and angular springs. To model the non-bonded attractive interaction between two subunits, we introduced the standard 12/6 Lennard-Jones $(\mathrm{L})$ potential energy $\left(V_{(r)}\right)$ between the beads on one end of a subunit and the corresponding beads on the end of the adjacent subunit (red dashed lines in Fig. 6b) as

$V_{(r)}=\varepsilon\left[\left(\frac{r_{m}}{r}\right)^{12}-2\left(\frac{r_{m}}{r}\right)^{6}\right]$

where $\varepsilon$ represents the depth of the potential well; $r$ represents the distance between two beads; and $r_{m}$ represents the force equilibrium distance. To introduce shear rigidity into the interface, as would be induced by backbone hydrogen bonds, we added pure repulsive potentials between alternating layers of beads (diagonal blue dashed lines in Fig. $6 \mathrm{~b}){ }^{31}$ The strength of the cohesive energy $\left(U_{c c}\right)$ between two subunits varies proportionally with the depth of the $12 / 6 \mathrm{LJ}$ potential well $\left(U_{c c}=9.36 \varepsilon, \varepsilon \in[0 \mathrm{kT}, 200 \mathrm{kT}]\right)$. The substrate is modeled by an explicit rectangular lattice, with distance between closest beads being $1 \mathrm{~nm}$ along the nanofiber's axis direction and $0.5 \mathrm{~nm}$ along the other direction. The substrate is fixed and the $12 / 6 \mathrm{LJ}$ potential between substrate beads are excluded. The adhesive energy between the subunit and the substrate was also simulated using the $12 / 6 \mathrm{~L}$ potential, with an force equilibrium distance of $1 \mathrm{~nm}$. Its strength also varies proportionally with the depth of the $12 / 6 \mathrm{~L}$ potential well $\left(U_{c s}=85.1 \varepsilon, \varepsilon \in[0 \mathrm{kT}, 2 \mathrm{kT}]\right)$.

Visual Molecular Dynamics (VMD) was used to visualize models, ${ }^{66}$ and simulations were carried out using LAMMPS (Large-scale Atomic/Molecular Massively Parallel Simulator). ${ }^{67}$ We first minimized the system energy for 100,000 steps and then performed the equilibration simulations for $1.5 \mathrm{~ns}$. The timestep used is $15 \mathrm{fs}$. After equilibrating the nanofiber on the substrate, we peeled the nanofiber by moving the first unit of the nanofiber at a constant velocity $(0.68 \mathrm{~m} / \mathrm{s})$ using steered molecular dynamics (SMD) in the $z$-axis direction (normal to the substrate), which prevented sliding of the nanofiber along the substrate. To calculate the cohesive energy, we minimized the system of two adjacent subunits for 100,000 steps and performed the equilibration simulations for $1 \mathrm{~ns}$. Then we calculated the nonbonded energy between the two subunits. The same procedure was applied to calculate the nonbonded energy between one subunit and the substrate. All simulations were conducted using the canonical ensemble (NVT) with Nose-Hoover thermostat at $300 \mathrm{~K}$. The temperature damping parameter was chosen as $15 \mathrm{ps}$.

\section{DATA AVAILABILITY}

The data that support the findings of this study are available from the corresponding author upon reasonable request.

\section{ACKNOWLEDGEMENTS}

This research was sponsored by an award from the Office of Naval Research Young Investigator Program (grant \#N00014-15-1-2701). The authors acknowledge a supercomputing grant from the Northwestern University High Performance Computing Center and the Department of Defense Supercomputing Resource Center. We also thank Elizabeth DeBenedictis and Yao Zhang for their helpful comments on improving this work. 


\section{AUTHOR CONTRIBUTIONS}

S.K. contributed to the design of the research. S.K. and A.W. contributed to the implementation of the research, to the analysis of the results and to the writing of the manuscript.

\section{ADDITIONAL INFORMATION}

Competing interests: The authors declare no competing interests.

Publisher's note: Springer Nature remains neutral with regard to jurisdictional claims in published maps and institutional affiliations.

\section{REFERENCES}

1. Larsen, P. et al. Amyloid adhesins are abundant in natural biofilms. Environ. Microbiol. 9, 3077-3090 (2007).

2. Berne, C., Ellison, C. K., Ducret, A. \& Brun, Y. V. Bacterial adhesion at the single-cell level. Nat. Rev. Microbiol 16, 616-627 (2018).

3. Koli, P., Sudan, S., Fitzgerald, D., Adhya, S. \& Kar, S. Conversion of commensa Escherichia coli K-12 to an invasive form via expression of a mutant histone-like protein. MBio 2, e00182-00111 (2011).

4. Prigent-Combaret, $C$. et al. Complex regulatory network controls initial adhesion and biofilm formation in Escherichia coli via regulation of thecsgD gene. $J$. Bacteriol. 183, 7213-7223 (2001).

5. Nasr, A., Olsén, A., Sjöbring, U., Müller-Esterl, W. \& Björck, L. Assembly of human contact phase proteins and release of bradykinin at the surface of curliexpressing Escherichia coli. Mol. Microbiol. 20, 927-935 (1996).

6. Herwald, H. et al. Activation of the contact-phase system on bacterial surfaces-a clue to serious complications in infectious diseases. Nat. Med. 4, 298 (1998).

7. Olsén, A. et al. Identification of two protein-binding and functional regions of curli, a surface organelle and virulence determinant of Escherichia coli. J. Biol. Chem. 277, 34568-34572 (2002).

8. Austin, J. W., Sanders, G., Kay, W. W. \& Collinson, S. K. Thin aggregative fimbriae enhance Salmonella enteritidis biofilm formation. FEMS Microbiol. Lett. 162, 295-301 (1998)

9. Pawar, D., Rossman, M. \& Chen, J. Role of curli fimbriae in mediating the cells of enterohaemorrhagic Escherichia coli to attach to abiotic surfaces. J. Appl. Microbiol. 99, 418-425 (2005).

10. Li, C., Adamcik, J. \& Mezzenga, R. Biodegradable nanocomposites of amyloid fibrils and graphene with shape-memory and enzyme-sensing properties. Nat. Nanotechnol. 7, 421 (2012).

11. Li, C., Bolisetty, S. \& Mezzenga, R. Hybrid nanocomposites of gold single-crystal platelets and amyloid fibrils with tunable fluorescence, conductivity, and sensing properties. Adv. Mater. 25, 3694-3700 (2013).

12. Li, C. et al. Amyloid-hydroxyapatite bone biomimetic composites. Adv. Mater. 26 3207-3212 (2014).

13. Nguyen, P. Q., Botyanszki, Z., Tay, P. K. R. \& Joshi, N. S. Programmable biofilmbased materials from engineered curli nanofibres. Nat. Commun. 5, 4945 (2014).

14. Zhong, $C$. et al. Strong underwater adhesives made by self-assembling multiprotein nanofibres. Nat. Nanotechnol. 9, 858 (2014).

15. Zhang, T., Yuk, H., Lin, S., Parada, G. A. \& Zhao, X. Tough and tunable adhesion of hydrogels: experiments and models. Acta Mech. Sin. 33, 543-554 (2017).

16. Yuk, H., Zhang, T., Lin, S., Parada, G. A. \& Zhao, X. Tough bonding of hydrogels to diverse non-porous surfaces. Nat. Mater. 15, 190 (2016).

17. Meredith, H. J., Jenkins, C. L. \& Wilker, J. J. Enhancing the adhesion of a biomimetic polymer yields performance rivaling commercial glues. Adv. Funct. Mater. 24, 3259-3267 (2014).

18. North, M. A., Del Grosso, C. A. \& Wilker, J. J. High strength underwater bonding with polymer mimics of mussel adhesive proteins. ACS Appl. Mater. Interfaces 9 7866-7872 (2017).

19. Dorval Courchesne, Nm-M., Duraj-Thatte, A., Tay, P. K. R., Nguyen, P. Q. \& Joshi, N. $S$. Scalable production of genetically engineered nanofibrous macroscopic materials via filtration. ACS Biomater. Sci. Eng. 3, 733-741 (2016).

20. Knowles, T. P. \& Buehler, M. J. Nanomechanics of functional and pathological amyloid materials. Nat. Nanotechnol. 6, 469 (2011).

21. Jain, N. et al. Inhibition of curli assembly and Escherichia coli biofilm formation by the human systemic amyloid precursor transthyretin. Proc. Natl Acad. Sci. 114, 12184-12189 (2017).

22. Qi, Q., Zhao, T.-X., An, B.-L., Liu, X.-Y. \& Zhong, C. Self-assembly and morphological characterization of two-component functional amyloid proteins. Chin. Chem. Lett. 28, 1062-1068 (2017).

23. Kalyoncu, E., Ahan, R. E., Olmez, T. T. \& Seker, U. O. S. Genetically encoded conductive protein nanofibers secreted by engineered cells. RSC Adv. 7, 32543-32551 (2017).
24. Barnhart, M. M. \& Chapman, M. R. Curli biogenesis and function. Annu. Rev. Microbiol. 60, 131-147 (2006).

25. Evans, M. L. \& Chapman, M. R. Curli biogenesis: order out of disorder. Biochim. Et. Biophys. Acta 1843, 1551-1558 (2014).

26. Sleutel, M. et al. Nucleation and growth of a bacterial functional amyloid at single-fiber resolution. Nat. Chem. Biol. 13, 902 (2017).

27. Oh, Y. J. et al. Curli mediate bacterial adhesion to fibronectin via tensile multiple bonds. Sci. Rep. 6, 33909 (2016).

28. Tian, P. et al. Structure of a functional amyloid protein subunit computed using sequence variation. J. Am. Chem. Soc. 137, 22-25 (2014).

29. DeBenedictis, E., Ma, D. \& Keten, S. Structural predictions for curli amyloid fibril subunits CsgA and CsgB. RSC Adv. 7, 48102-48112 (2017).

30. DeBenedictis, E. P., Liu, J. \& Keten, S. Adhesion mechanisms of curli subunit CsgA to abiotic surfaces. Sci. Adv. 2, e1600998 (2016).

31. Zhang, Y., Wang, A., DeBenedictis, E. P. \& Keten, S. Bending energy penalty enhances the adhesive strength of functional amyloid curli to surfaces. Nanotechnology 28, 464002 (2017)

32. Chen, H., Feng, X., Huang, Y., Huang, Y. \& Rogers, J. A. Experiments and viscoelastic analysis of peel test with patterned strips for applications to transfer printing. J. Mech. Phys. Solids 61, 1737-1752 (2013).

33. Kim, K. S. \& Aravas, N. Elastoplastic analysis of the peel test. Int. J. Solids Struct. 24 417-435 (1988).

34. Suo, Z. \& Hutchinson, J. W. Interface crack between two elastic layers. Int. J. Fract 43, 1-18 (1990).

35. Gao, E. et al. Mechanical exfoliation of two-dimensional materials. J. Mech. Phys. Solids 115, 248-262 (2018).

36. He, L., Yan, S., Li, B. \& Chu, J. Directional adhesion behavior of a single elastic fiber. J. Appl. Phys. 112, 013516 (2012).

37. Mojdehi, A. R., Holmes, D. P. \& Dillard, D. A. Friction of extensible strips: an extended shear lag model with experimental evaluation. Int. J. Solids Struct. 124, 125-134 (2017).

38. Peng, Z. \& Chen, S. The effect of geometry on the adhesive behavior of bioinspired fibrils. Soft Matter 8, 9864-9869 (2012).

39. Peng, Z., Chen, S. \& Soh, A. Peeling behavior of a bio-inspired nano-film on a substrate. Int. J. Solids Struct. 47, 1952-1960 (2010).

40. Ponce, S., Bico, J. \& Roman, B. Effect of friction on the peeling test at zerodegrees. Soft Matter 11, 9281-9290 (2015).

41. Pugno, N. M. The theory of multiple peeling. Int. J. Fract. 171, 185-193 (2011).

42. Putignano, C., Afferrante, L., Mangialardi, L. \& Carbone, G. Equilibrium states and stability of pre-tensioned adhesive tapes. Beilstein J. Nanotechnol. 5, 1725 (2014).

43. Qian, J., Lin, J., Xu, G.-K., Lin, Y. \& Gao, H. Thermally assisted peeling of an elastic strip in adhesion with a substrate via molecular bonds. J. Mech. Phys. Solids 101 197-208 (2017).

44. Kierfeld, J. Force-induced desorption and unzipping of semiflexible polymers. Phys. Rev. Lett. 97, 058302 (2006).

45. Seifert, U. \& Lipowsky, R. Adhesion of vesicles. Phys. Rev. A 42, 4768 (1990).

46. Paul, T. J. et al. Structural and mechanical properties of amyloid beta fibrils: a combined experimental and theoretical approach. J. Phys. Chem. Lett. 7, 2758-2764 (2016).

47. Adamcik, J. et al. Understanding amyloid aggregation by statistical analysis of atomic force microscopy images. Nat. Nanotechnol. 5, 423 (2010).

48. Jordens, S., Isa, L., Usov, I. \& Mezzenga, R. Non-equilibrium nature of twodimensional isotropic and nematic coexistence in amyloid fibrils at liquid interfaces. Nat. Commun. 4, 1917 (2013).

49. Choi, B., Yoon, G., Lee, S. W. \& Eom, K. Mechanical deformation mechanisms and properties of amyloid fibrils. Phys. Chem. Chem. Phys. 17, 1379-1389 (2015).

50. Choi, H., Lee, M., Park, H. S. \& Na, S. The effect of structural heterogeneity on the conformation and stability of $\mathrm{A} \beta$-tau mixtures. RSC Adv. 6, 52236-52247 (2016).

51. Kim, J., Lee, M., Baek, I., Yoon, G. \& Na, S. The mechanical response of hIAPP nanowires based on different bending direction simulations. Phys. Chem. Chem. Phys. 16, 18493-18500 (2014).

52. Tran, T.-T., Nguyen, P. H. \& Derreumaux, P. Alzheimer's disease: insights into amyloid fibril formation from lattice monte carlo simulations. Biophys. J. 110, 219a (2016).

53. Xiao, Y. et al. $A \beta(1-42)$ fibril structure illuminates self-recognition and replication of amyloid in Alzheimer's disease. Nat. Struct. Mol. Biol. 22, 499 (2015).

54. Gazit, E. A possible role for $\pi$-stacking in the self-assembly of amyloid fibrils. FASEB J. 16, 77-83 (2002)

55. Röder, K. \& Wales, D. J. Energy landscapes for the aggregation of A $\beta 17-42$. J. Am. Chem. Soc. 140, 4018-4027 (2018).

56. Yoon, G., Kwak, J., Kim, J. I., Na, S. \& Eom, K. Mechanical characterization of amyloid fibrils using coarse-grained normal mode analysis. Adv. Funct. Mater. 21, 3454-3463 (2011). 
57. Yoon, G., Lee, M., Kim, J. I., Na, S. \& Eom, K. Role of sequence and structural polymorphism on the mechanical properties of amyloid fibrils. PLOS ONE 9, e88502 (2014).

58. Knowles, T. P. et al. Role of intermolecular forces in defining material properties of protein nanofibrils. Science 318, 1900-1903 (2007).

59. $\mathrm{Hu}, \mathrm{B}$. et al. Polyphenol-binding amyloid fibrils self-assemble into reversible hydrogels with antibacterial activity. ACS nano 12, 3385-3396 (2018).

60. Jenkins, C. L., Meredith, H. J. \& Wilker, J. J. Molecular weight effects upon the adhesive bonding of a mussel mimetic polymer. ACS Appl. Mater. Interfaces 5, 5091-5096 (2013).

61. Matos-Pérez, C. R., White, J. D. \& Wilker, J. J. Polymer composition and substrate influences on the adhesive bonding of a biomimetic, cross-linking polymer. J. Am. Chem. Soc. 134, 9498-9505 (2012).

62. Xuan, W. et al. Site-specific incorporation of a thioester containing amino acid into proteins. ACS Chem. Biol. 13, 578-581 (2018)

63. Heinzmann, C., Weder, C. \& de Espinosa, L. M. Supramolecular polymer adhesives: advanced materials inspired by nature. Chem. Soc. Rev. 45, 342-358 (2016).

64. Pfeifer, W., Lill, P., Gatsogiannis, C. \& Saccà, B. Hierarchical assembly of DNA filaments with designer elastic properties. ACS nano 12, 44-45 (2017).

65. Marshall, K. E. et al. Hydrophobic, aromatic, and electrostatic interactions play a central role in amyloid fibril formation and stability. Biochemistry 50, 2061-2071 (2011).
66. Humphrey, W., Dalke, A. \& Schulten, K. VMD: visual molecular dynamics. J. Mol. Graph. 14, 33-38 (1996).

67. Plimpton, S. Fast parallel algorithms for short-range molecular dynamics. J. Comput. Phys. 117, 1-19 (1995).

Open Access This article is licensed under a Creative Commons Attribution 4.0 International License, which permits use, sharing, adaptation, distribution and reproduction in any medium or format, as long as you give appropriate credit to the original author(s) and the source, provide a link to the Creative Commons license, and indicate if changes were made. The images or other third party material in this article are included in the article's Creative Commons license, unless indicated otherwise in a credit line to the material. If material is not included in the article's Creative Commons license and your intended use is not permitted by statutory regulation or exceeds the permitted use, you will need to obtain permission directly from the copyright holder. To view a copy of this license, visit http://creativecommons. org/licenses/by/4.0/.

(c) The Author(s) 2019 